\title{
ITIL Incident Management Process Reengineering in Industry 4.0 Environments
}

\author{
Jiqing $\mathrm{CaO}^{1, \text { a }}$, Shuhai Zhang ${ }^{2, \mathrm{~b}}$ \\ ${ }^{1}$ Department of Information Engineering, Suzhou Industrial Park Institute of Service Outsourcing \\ ${ }^{2}$ Department of IT Service, Bosch Automotive Products (Suzhou) Co., Ltd. \\ aemail: george.cao@siso.edu.cn, bemail:zhangsh@tom.com
}

Keyword: Industry 4.0; ITIL; DevOps; Incident Management Process; Process Improvement

\begin{abstract}
The new technologies such as virtual resources and RFID in Industry 4.0 make traditional ITIL Incident Management Process can not adapt to the new environment anymore. Based Agile and Lean thinking of DevOps, this paper improved and designed on the traditional ITIL Incident Management Processes, proposed the use of automated procedures to integrate multi-step activities in the old process, added new incident monitoring and processing procedures based on new technologies such as virtual resources and RFID, proposed to break the different functions split, and establish a unified DevOps incident support team. The new process well guided the incident management works for the joint venture, and greatly improved the efficiency of its troubleshooting.
\end{abstract}

\section{Introduction}

Industry currently has just set off a wave of version 4.0. There are still no mature operation and maintenance solutions and products for industry 4.0 based on complex and large-scale system and new technologies, and the traditional ITIL processes cannot adapt to these new conditions. Focus on new technology environment of Industry 4.0, with the Agile and Lean thinking of operation and maintenance of DevOps (Development and Operation), this article improved and designed the new process on the traditional ITIL Incident Management Process, and provided valuable reference to the joint venture.

\section{ITIL Incident Management Process}

ITIL (IT Infrastructure Library) is currently the international de facto standard for IT operations. ITIL describe the "incident" as "Unexpected IT service disruption or Degradation of IT service quality. Configure Item has not been an impact on the service failure is also an incident, such as a node in clusters goes down.” The Incident Management Process of ITIL is a process for handling all incidents that may be reported from varies of way and has been known thousands of businesses successfully managed IT system failures in the past decade.

ITIL Incident Management Process (see Figure 1) comprising the steps of: Source of Incident, Incident Confirmation, Incident Records, Incident Classification, Incident Priority, Initial Diagnosis, Escalation Judgment, Investigation and Diagnosis, Resolution and Recovery, Incident Close. [1] 


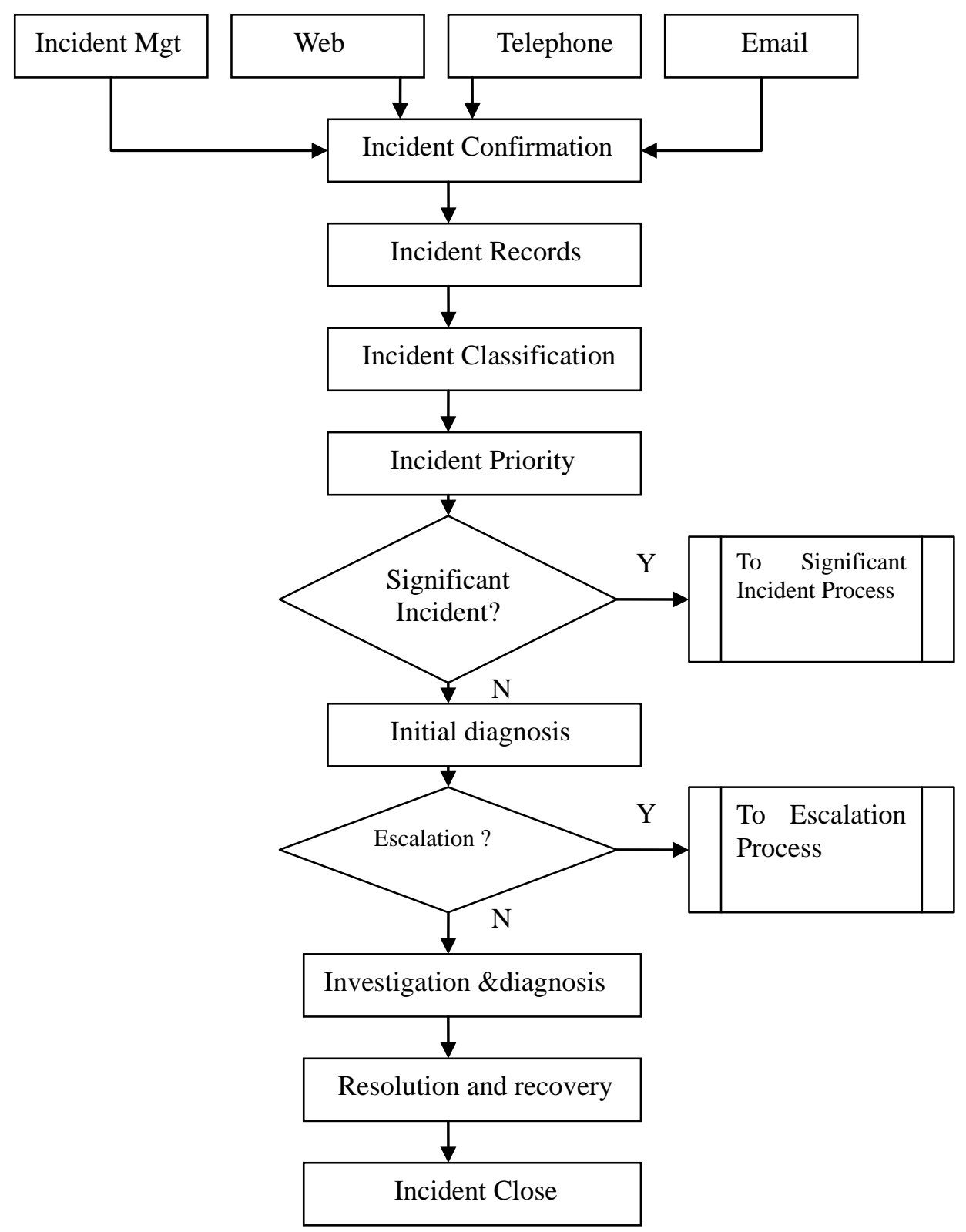

Fig.1. ITIL Incident Management Process

This process contains the activities of up to eleven steps and involves multiple roles and functions such as support, technology, quality and business, so it would have quite a few time delays, and so reduce the efficiency of the incident treatment. In the same time, it has still not considered the impact of new technologies such as virtual resources on the process.[2]

\section{The Impact of Industry 4.0 Environment to Incident Management Process}

Currently, there is still no final standard such as the data exchange protocol for Industry 4.0, but the involved technologies and architecture is recognized, that is, the Industry 4.0 is based on a distributed network of information technology to all aspects of the organic integration of industrial processes, these IT technologies include cloud computing, Internet of things, mobile Internet, big data, virtual reality, artificial intelligence and network security. ${ }^{[3]}$

The new technologies in Industry 4.0 will bring the following changes in the traditional ITIL Incident Management Processes:

3.1 Virtual Resources

(1) Consider virtual resources in the Incident Sources and Classification

In addition to the physical resources and applications in the Incident sources and classification, virtualization also introduces new resources, such as virtual clusters, virtual machines, virtual 
networks, virtual switches, virtual data storage, which will be related to the Incident monitoring, reporting and resolving manner.

(2) Consider virtual resource indicators in Incident Monitoring

In addition to the indicators of original physical resources and applications, it also must consider the key indicators of variety of the virtual resources, such as virtual CPU usage, virtual memory usage, virtual disk I/O rates and virtual network packet loss rate, etc., to detect anomalies and to take proactive actions.

(3) New way of the Incident Resolution for virtual resources

Virtual resources support self-service application of resources, expansion, recovery and automatic transfer. When an incident occurs, it can not only achieve rapid deployment, and dynamically add computing resources as needed when the application is busy, release computing resources when the application is idle, but also can make the dynamic migration of computing resources in the resource pool. Different from the need to preserve the host operating environment and save the software installation package in the traditional environment, a virtual machine image file loaded at any time means new virtual device can quickly enter the state of production when needed. [4]

3.2 Other New Technologies

(1) RFID (Radio Frequency Identification) technology makes some hardware failures anywhere be reported timely to the system and handled efficiently. Artificial intelligence technology can greatly improve the hardware fault processing efficiency, such as failure to report with RFID, smart car and even the UAV(Unmanned Aerial Vehicle) can precisely transported the replaceable resources to the incident site, install and deploy automatically by the robot.

(2) Big Data technology makes troubleshooting is no longer a stop-gap, but may be predictive in nature, so the system can take appropriate preventive measures before failure really happened. This can be a new way of incident monitoring.

(3) Virtual Reality can display failures and alarms directly on the System topology, helping the support engineer easily handle and close the incidents by automated scripts.

(4) In addition to technical factors, Industrial 4.0 demands for faster incident processing speed, to ensure continuity and availability of services. And the industrial network security requirements for the new resource for security and cooperation regulatory treatment before they be on-line, so as to ensure the system's safety and security [5]. As we can see, the traditional ITIL Incident Management Process is difficult to adapt to the new industry 4.0 environment.

\section{DevOps Operation and Maintenance View}

DevOps represents the latest view of Agile and Lean for the current operation and maintenance management. It is the new operation and maintenance mode proposed in the background of the new technologies such as cloud computing and automated operation, emphasizing automation and ongoing operation and maintenance of everything. DevOps propose to break the previous segmentation among the different functions, promote support engineer involved in the technical development process and the developer involved in the operation and maintenance process, and establish a unified support team. [6]

The Industry 4.0 grade huge number of devices in the system, the system is complex and across different regions and organizations, incidents to resolve may involve different departments and even organizations, such as technical, operational and maintenance. So if you want the large-scale system has the efficient operation and maintenance, it is necessary to implement the agile and lean thinking of DevOps, and use the cloud computing and automation tools and other technologies to achieve incident automated processing, to establish the unified support team of cross-technology, business and operations of different departments, to minimize delay in the transfer process flow, thereby improving the efficiency of the incident management and meet the operation requirements of Industrial 4.0. 


\section{Incident Management Process Improvements and Design}

By analyzing the influencing factors on the traditional ITIL Incident Management Process, this paper improves and designs the new Incident Management Process Based on the Agile and Lean thinking. The new process includes four modules of Incident Monitoring, Incident Acceptance, Incident Treatment and Incident Management as the life cycle of troubleshooting process. For a small part can not be done through automation, nor directly related to the special requirements of manufacturing business failures, the new process still retains the traditional processes as a branch, see Figure 2.

5.1 Incident Monitoring Module

Incident Monitoring Module adds support for virtual resources as a new incident object, and adds the new automated monitoring methods based on virtual resources, Big Data and RFID technologies. With respect to the original process, when each of the incidents is reported, this module completes the "Incident Acknowledgment, Classification and Priority" and other multi-step activities of the traditional process in accordance with defined criteria.

5.2 Incident Acceptance Module

This Module is responsible for the integration treatment for the multi-step activities of "Incident Record, Classify, Service Requests Judgment, and Major Incident Judgment" of the traditional process for the incidents reported from Incident Monitoring Module.

5.3 Incident Treatment Module

This Module designs the corresponding incident treatment flow branches for the technologies of virtual resources, RFID and virtual reality, each branch also includes some sub-modules. Increased the Virtual Resource Management Sub-module, which is responsible for managing the configuration of virtual resources and indicators; Increased the New Device Management Sub-module, which is responsible for warehouse queries and other functions of the replaceable equipments for the corresponding incident reported from RFID; Increased the Distribution Management Sub-module, which is responsible for automated distribution with artificial intelligence technology; Increased Virtual Resource Monitoring Sub-module, which is responsible for monitoring virtual resources performance and capacity and other indicators; Increased Security Management Sub-module to check whether there is a new virtual resource security risks before going on-line; Increased the Automated Scripting Sub-module, which is responsible for the automated incident solutions through manual intervention triggered scripts in the virtual reality topology; Increased Resources On-line Sub-module, which is responsible for deployment, testing and validation of the resources. [7]

5.4 Incident Management Module

Incident Management Module is not listed in Figure 2, this module is mainly responsible for the incident query, reporting and customization.

5.5 DevOps Unified Incident Support Team.

Efficient process also requires an efficient support team. It is a cross-sectoral, or even across the organization DevOps unified incident support team. The team master all the skills required for incident treatment so that it can reduces or even eliminates the transfer delay across different departments or organizations in the process, and greatly improving the efficiency of incident treatment. 


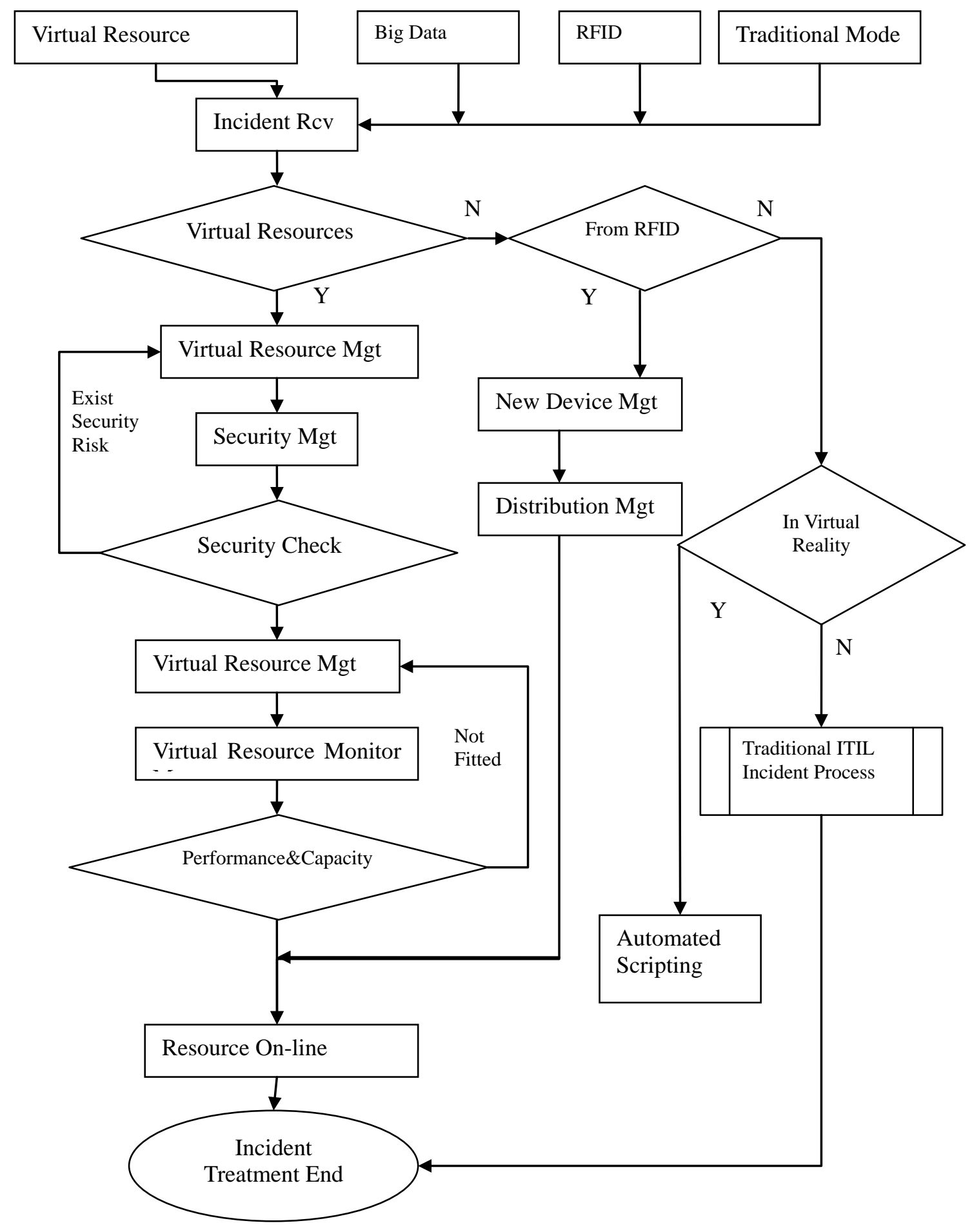

Fig.2. The New Incident Management Process

\section{Summaries}

The study comes from the practice of the needs our joint venture. They design and construct the Incident Management Module of the Cloud Maintenance Platform for their Industry 4.0 system based on this research. They use automated procedures to achieve the multi-step activities in the traditional process, achieve the incident management for the virtual resources automated; They achieve troubleshooting for key hardware of the production line automated with RFID, Andon System, Warehouse Management System and Smart Car System; They achieve application troubleshooting using the automated scripts program in virtual reality system.

With the rapid advance of Industry 4.0, the new process proposed in this study will provide 
valuable assistance for Incident Management for the implementation of new-comer Industry 4.0 enterprises.

\section{Acknowledgement}

In this paper, the research was sponsored by the Service Outsourcing Foundation of Suzhou Institute Park Service Outsourcing Institute (Project No. ky-xjy02).

\section{References}

[1]Service_operation.ITIL Version 3 (2011):72-86

[2]Chen Chunhua, Li Jun.Liang Huan,Yao Wensheng. IT Service Management Implementation Strategy of Resource Pool Environment[J]. Telecommunications Science, 2012(9):144-146

[3]DKE/DIN. The German Standardization Roadmap Industrie 4.0[R]. VDE ASSOCIATION FOR ELECTRICAL, ELECTRONIC \& INFORMATION TECHNOLOGIES,2014.11

[4]Chen Chunhua, Li Jun.Liang Huan,Yao Wensheng. IT Service Management Implementation Strategy of Resource Pool Environment[J]. Telecommunications Science, 2012(9) :144-145

[5]Wang Lingyun, Lihui, The New Requirement for the equipment management of Industry 4.0[J]. China Plant Engineering 2015(7):2-3

[6]JAMES ROCHE. Adopting DevOps Practices in Quality Assurance[J].Communications of the ACM, 2013(11):3-5

[7]Cao Jian. Analysis of IT operation based on Virtual background [J]. Information \& Communications, 2015(8):154-155 